This document is the Accepted Manuscript version of a Published Work that appeared in final form in Macromolecules 52(18) : 6904-6912 (2019), copyright @ 2019 American Chemical Society after peer review and technical editing by the publisher. To access the final edited and published work see https://doi.org/10.1021/acs.macromol.9b00542

\title{
Segmental Dynamics Govern the Cold Crystallization of Poly(lactic acid)
}

\section{in Nanoporous Alumina}

\author{
Guangyu Shi $^{\dagger, \ddagger}$, Yu Guan ${ }^{\dagger, \ddagger}$, Guoming Liu*, ${ }^{\dagger, \ddagger}$, Alejandro J. Müller ${ }^{\S, \|}$, and Dujin Wang ${ }^{\dagger, \ddagger}$
}

${ }^{\dagger}$ Beijing National Laboratory for Molecular Sciences (BNLMS), CAS Key Laboratory of Engineering Plastics, CAS

Research/Education Center for Excellence in Molecular Sciences, Institute of Chemistry, Chinese Academy of Sciences, Beijing 100190, China

${ }^{\ddagger}$ University of Chinese Academy of Sciences, Beijing 100049, China

$\S$ POLYMAT and Polymer Science and Technology Department, Faculty of Chemistry, University of the Basque Country UPV/EHU, Paseo Manuel de Lardizabal 3, 20018 Donostia-San Sebastián, Spain

" IKERBASQUE, Basque Foundation for Science, Bilbao, Spain

* Corresponding author: gmliu@iccas.ac.cn 


\section{ABSTRACT}

How segmental mobility influences the crystallization behavior of polymers in confined spaces is still not fully understood. In the present work, a systematic study of the segmental dynamics was carried out by dielectric relaxation spectroscopy (DRS) on racemic poly(DL-lactic acid) (PDLLA) confined in nanoporous alumina. The effect of pore size and thermal history were examined. Two glass transition temperatures $\left(T_{\mathrm{g}}\right)$ were observed, and an unusual "hysteresis" of the segmental relaxation time was detected. At lower temperatures, the segmental mobility of PDLLA "speeds up" firstly and "slows down" upon annealing. Both effects indicate the dynamically heterogeneous and nonequilibrium nature of chain segments under confinement. The "equilibrating process" of the relaxation time was monitored by DRS and the characteristic time exhibited Arrhenius behavior. The experimental evidence supports a reversible adsorption/desorption mechanism of chain segments. The enhanced cold crystallization of poly(lactic acid) (PLLA) we reported previously (Macromolecules 2015, 48 (8), 2526-2533) can be explained adequately with the "enhanced mobility” of segments. This was further justified by the shift of the cold crystallization temperature towards high temperatures of infiltrated PLLA after annealing.

\section{INTRODUCTION}

The dynamics of soft matter under nanoscale confinement differs from bulk behavior. The physical nature of the deviations has been attracting much attention in the past two decades. ${ }^{1,2}$ Model systems have been constructed and investigated extensively, in particular polymer thin films, ${ }^{3,4}$ and nanocomposites. ${ }^{5-7}$ Generally, depending on the choice of polymer and substrate, changes of segmental relaxation time or shifts in the glass transition temperatures (both increases or decreases with confinement) have been reported, which is usually discussed as a counterbalance of different factors. A free-surface or repulsive interface ${ }^{8-11}$ accelerates the segmental mobility ( $T_{\mathrm{g}}$ depression), while adsorptive or attractive interface ${ }^{2,12-14}$ generally exhibits a slowdown effect on the dynamics ( $T_{\mathrm{g}}$ increase).

Exploring the effect of 2D confinement on the segmental dynamics of polymers is a very interesting subject. Anodic aluminum oxide (AAO) membranes with arrays of isolated, parallel, uniform nanocylinders have become a model system for studying the effects of confinement on the segmental or chain dynamics of amorphous or semicrystalline polymers. Many polymers, such as poly(styrene) (PS), ${ }^{15}$ PMMA, ${ }^{16-19}$ polysiloxanes, ${ }^{14,}{ }^{20}$ cis-1,4polyisoprene (PI), ${ }^{21}$ polycarbonate (PC), ${ }^{22}$ poly(vinylidene fluoride) (PVDF), ${ }^{23}$ poly(propylene glycol) (PPG), ${ }^{24,25}$ and polypeptides ${ }^{26}$ have been investigated. 
Despite the variety of studies, a general understanding of polymer dynamics within AAO templates has not been reached so far, as the size dependence of the dynamics does not always show clear trends. The speedup of segmental dynamics (decrease of $T_{\mathrm{g}}$ ) has been observed in a variety of amorphous polymers, such as PMPS, ${ }^{20}$ poly(vinyl acetate) (PVAc),${ }^{20}$ 1,4-polybutadiene (PB), ${ }^{20}$ poly(dimethylsiloxane) (PDMS), ${ }^{20} \mathrm{PMMA}^{19}$ and $\mathrm{PPG}^{24}$, as well as in several semi-crystalline polymers, such as PCL ${ }^{27}$. However, a rather irregular change of the segmental dynamics has been frequently observed. For instance, the segmental relaxation time of PBLG polypeptide does not change monotonically with pore size. ${ }^{26}$ Blaszczyk-Lezak et al. ${ }^{16}$ reported that the PMMA within $28 \mathrm{~nm}$ diameter AAO showed opposite trends as compared with the samples infiltrated in 35 and $65 \mathrm{~nm}$ AAO. ${ }^{16}$ In cis-1,4-polyisoprene, the $T_{\mathrm{g}}$ was reported to be invariant upon confinement, but the width of the segmental and the chain relaxation peaks broadened remarkably. ${ }^{21}$ A similar broadening effect was seen in poly(ethylene oxide) (PEO) and the change of average segmental relaxation time did not show a clear trend..$^{28}$ Broadening and decreased $T_{\mathrm{g}}$ value was also observed in polycarbonate. ${ }^{22}$ Such broadening is frequently explained as a manifestation of retarded global mobility. ${ }^{21}$ Decoupling of dynamic and thermal $T_{\mathrm{g}}$ seems to be a general phenomenon in confined systems. ${ }^{29}$ The chain mobility has also been evaluated by indirect means, i.e., the density of entanglements has been found to decrease with confinement by small-angle neutron scattering (SANS) ${ }^{15}$ and computer simulation. ${ }^{14}$ Serghei et al. ${ }^{30}$ found no variations in the segmental dynamics of P2VP within nanopores.

It is believed that there is an immobilized layer in the proximity of the pore surface when PDMS and PEO are infiltrated in AAO templates. ${ }^{14}$ In some cases, more than one glass transition temperatures have been observed, ${ }^{17,} 18$ which naturally leads to a "multi-layer model". Two different $T_{\mathrm{g}} \mathrm{s}$ are detected in infiltrated PMMA within $80 \mathrm{~nm}$ AAO pores after slow cooling the melt, while only one is detected after fast cooling from the melt. ${ }^{17}$ It was anticipated that two distinct layers are formed during vitrification under slow cooling: an interfacial layer with a higher $T_{\mathrm{g}}$ and a core with a lower $T_{\mathrm{g}}$, as compared with the bulk. Fast cooled PMMA showed a single $T_{\mathrm{g}}$. Three $T_{\mathrm{g}} \mathrm{S}$ were observed for infiltrated PMMA within larger pores $\left(300 \mathrm{~nm}\right.$ ), and a three-layer model was further proposed. ${ }^{18}$ Similar two $T_{\mathrm{g} S}$ were found in $\mathrm{PPG}^{24,25}$, the times for segmental relaxation and normal mode increased below a crossover temperature. These phenomena highlighted the role of thermal history on the dynamics in confined systems.

It is anticipated that segmental dynamics should have a strong influence on crystallization under confinement. It is known that the crystallization rate is determined by nucleation and growth, with segmental mobility playing a role. However, because the nucleation mechanism variation usually has a more pronounced influence on crystallization rate, the effect of segmental dynamics is seldom demonstrated clearly. In our previous study, we showed an unusual acceleration effect of the crystallization rate when infiltrated PLLA was crystallized from the 
glassy state within AAO template, ${ }^{31}$ however it is still not known why the nucleation was promoted by the amorphous AAO wall. To address this issue, in this work, to eliminate the influence of crystallization, a racemic poly(lactic acid) (PDLLA) was selected. The segmental dynamics of PDLLA under confinement in AAO templates were systematically examined by dielectric relaxation spectroscopy (DRS). An acceleration effect of the segmental dynamics was observed. Interestingly, annealing leads to a slowdown effect on segmental dynamics. The results demonstrate a clear counterbalance of confinement and adsorption effects. Along with the slowdown of segmental dynamics during annealing, the cold crystallization rate of infiltrated PLLA decreased remarkably with annealing. Therefore, a strong correlation between the segmental mobility and the cold crystallization of PLLA was established.

\section{EXPERIMENTAL SECTION}

\subsection{Materials and Sample Preparation.}

Poly(DL-lactic acid) (PDLLA) with equimolar D and L isomers, with a number-average molecular weight $\left(M_{\mathrm{n}}\right)$ of 20,000 g/mol and a polydispersity index of 1.34 and hydroxyl-terminated poly(L-lactic acid) (PLLA) with a $M_{\mathrm{n}}$ of 7,000 g/mol and polydispersity index of 1.36 were purchased from Daigang Biomaterials Inc. (Jinan, China). AAO templates with pore diameters $\Phi=40 \mathrm{~nm}, 60 \mathrm{~nm}, 100 \mathrm{~nm}, 200 \mathrm{~nm}, 400 \mathrm{~nm}$, and a pore length of approximately 100 $\mu \mathrm{m}$, were purchased from Shanghai Shangmu Technology Co. Ltd. The preparation procedure has been published previously. 32,33

All the as-received empty AAO templates were rinsed with distilled waters and annealed at $773 \mathrm{~K}$ for 5 hours to get rid of the adsorbing water and organic molecules. The polymer samples were dried in a vacuum oven at $353 \mathrm{~K}$ overnight. The polymer films were placed on the surface of AAO in a home-made vacuum chamber and treated at $493 \mathrm{~K}$ under vacuum for 1.5 hours and then under nitrogen atmosphere for 1.5 hours. The residual polymer on the surface of AAO was cleaned completely according to our previous recommended procedure ${ }^{32}$.

\subsection{Characterization.}

The pores of AAO and morphology of the nanorods were studied by a scanning electron microscope (SEM, JSM-6700F JEOL). The acceleration voltage was $5 \mathrm{kV}$. To isolate the polymeric nanorods, the AAO template was solved in a phosphoric acid solution (3.5\% v/v) containing $4.5 \mathrm{~g} / \mathrm{mL}$ chromium trioxide.

Differential scanning calorimetry was utilized to investigate the crystallization/melting behavior of polymers. The instrument (DSC Q2000, TA) was calibrated before measurements. For cold crystallization from the glassy state, the samples were heated to $473 \mathrm{~K}$ to erase the thermal history. Subsequently, the samples were cooled to $273 \mathrm{~K}$ (100 
$\mathrm{K} / \mathrm{min}$ ). Finally, the samples were heated from 273 to $473 \mathrm{~K}$ at a heating rate of $10 \mathrm{~K} / \mathrm{min}$. To study the effect of annealing, the samples were first heated to $473 \mathrm{~K}$, then quenched to $342 \mathrm{~K}$ at $100 \mathrm{~K} / \mathrm{min}$. After annealing at $342 \mathrm{~K}$ for different times, the samples were heated to $473 \mathrm{~K}$ at $10 \mathrm{~K} / \mathrm{min}$.

Broadband dielectric spectroscopy (DRS) were measured using the Novacontrol Alpha dielectric spectrometer at ambient pressure. The complex dielectric permittivity $\varepsilon^{*}(\omega)=\varepsilon^{\prime}(\omega)-\mathrm{i} \varepsilon^{\prime \prime}(\omega)$ was recorded over $10^{-2} \sim 10^{7} \mathrm{~Hz}$. A Quatro Cryosystem was used to control the temperature. The bulk PDLLA was measured using a parallel plate cell (diameter: $20 \mathrm{~mm}$; gap: $0.2 \mathrm{~mm}$ ). AAO templates filled with PDLLA were measured using a similar sample cell (diameter: $20 \mathrm{~mm}$; thickness: 1 mm).

Three temperature protocols were used to perform the DRS measurements of the AAO infiltrated PDLLA. Protocol 1 (P1): first the thermal history was erased by heating to $473 \mathrm{~K}$, then the samples were cooled down to 213 $\mathrm{K}$ at a rate of $20 \mathrm{~K} / \mathrm{min}$. Then the frequency scan with a range of $10^{-2}$ to $10^{7} \mathrm{~Hz}$ was carried out isothermally at increasingly higher temperatures (i.e., every $2 \mathrm{~K}$ ). Each frequency scan takes about 30 minutes, therefore resulting in an equivalent heating rate of $\sim 0.067 \mathrm{~K} / \mathrm{min}$. Protocol 2 (P2): the frequency scan was performed from the melt, every $2 \mathrm{~K}$, with a frequency range of $10^{-2}$ to $10^{7} \mathrm{~Hz}$. Because each frequency scan under isothermal conditions requires 30 minutes, samples measured with protocol 2 (P2) have a very low cooling rate, $~ 0.067 \mathrm{~K} / \mathrm{min}$. Protocol 3 (P3): first the thermal history was erased by heating to $473 \mathrm{~K}$, then the samples were cooled down to $213 \mathrm{~K}$ at a rate of 20 $\mathrm{K} / \mathrm{min}$. Then temperature scans were performed during heating from the glassy state at a fixed frequency $(1 \mathrm{~Hz})$ and a heating rate of $\sim 3 \mathrm{~K} / \mathrm{min}$. Empty AAO templates were also measured by DRS using the same protocols and the signal was negligible as compared to the infiltrated AAO (Supporting Information Figure S1).

\section{RESULTS AND DISCUSSION}

\subsection{Morphology of PDLLA Nanorods.}

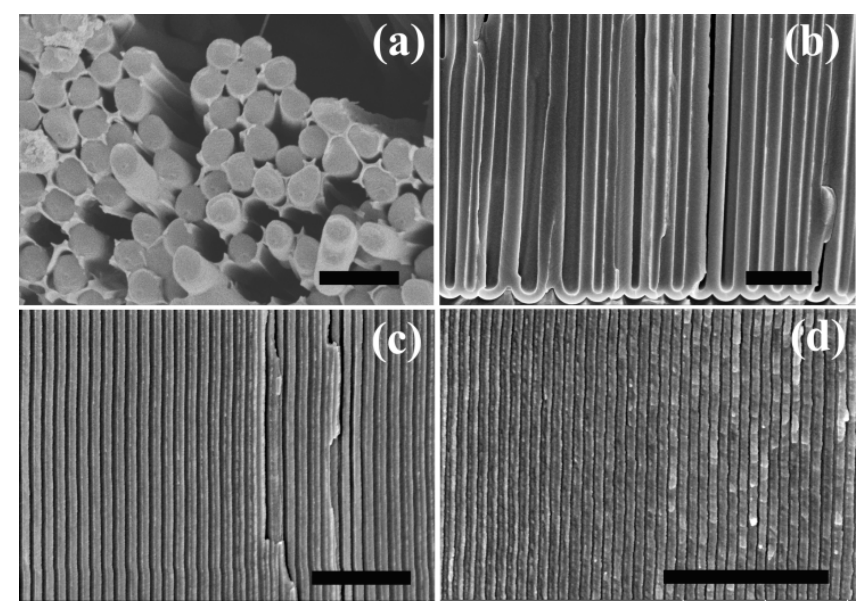


Figure 1. SEM images of PDLLA nanorods prepared with $400 \mathrm{~nm}$ AAO (a) and cross-sectional images of empty AAO with $\Phi=200$ (b), 100 (c) and $40 \mathrm{~nm}(\mathrm{~d})$. The length of the scale bars is $1 \mu \mathrm{m}$.

The nanorods of PDLLA and the cross-sectional morphology of AAO templates were shown in Figure 1. The pores of AAO exhibit uniform diameter. They are parallel to each other with no interconnections. The morphology indicates that the AAO membranes we employed provide an ideal model system to explore the physical behavior of polymers under confinement.

\subsection{Glass Transition Temperatures $\left(T_{\mathrm{g}}\right)$.}

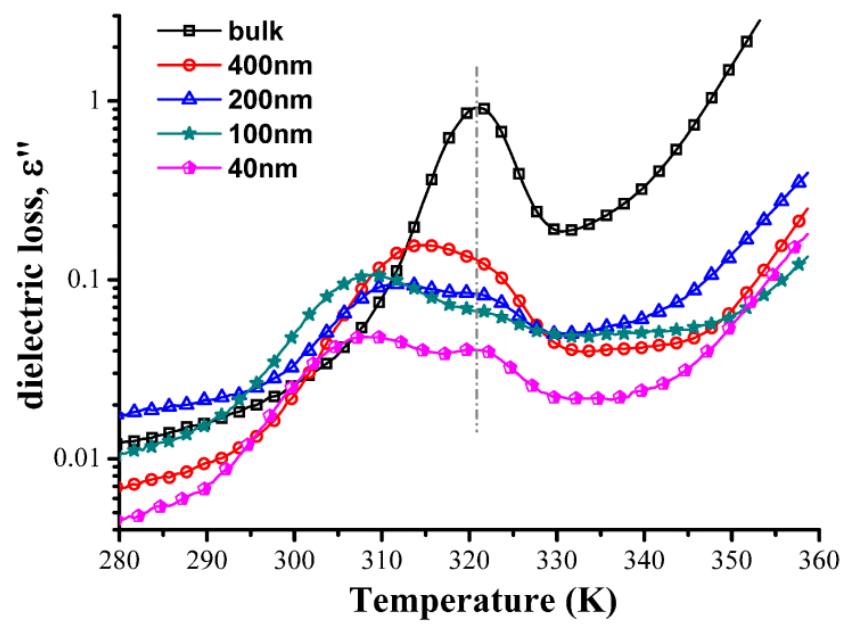

Figure 2. Dielectric loss ( $\left.\varepsilon^{\prime \prime}\right)$ curve of bulk and infiltrated PDLLA in different AAO templates. The samples were measured during heating (protocol P3) with a fixed frequency of $1 \mathrm{~Hz}$.

Because of the limited amount of polymers inside the AAO template, the DSC curves are too noisy to measure the glass transition. Fortunately, the DRS signal is much stronger. Figure 2 shows the $\varepsilon$ ” as a function of temperature during heating from the glassy state (at $\sim 3 \mathrm{~K} / \mathrm{min}$ ) after cooling the samples from the melt (473 $\mathrm{K}$ ) to $273 \mathrm{~K}$ at a cooling rate of $20 \mathrm{~K} / \mathrm{min}$.

The bulk PDLLA sample exhibits a single loss peak at $321 \mathrm{~K}$ in Figure 2, which corresponds to the local segmental relaxation $\left(T_{\mathrm{g}}\right)$ of PDLLA. The "normal mode” relaxation, corresponding to the fluctuation of the end-toend vector, is not observed in the experimental range because our sample has a high molecular weight. ${ }^{34,35}$ Two loss $^{3}$ peaks appear for the infiltrated PDLLA within smaller AAO pores. The loss peak of infiltrated PDLLA in 400 nm AAO is very broad and may be caused by a superposition of two peaks. The lower $T_{\mathrm{g}}$ locates at $\sim 308 \mathrm{~K}$ (13 K lower than the bulk $T_{\mathrm{g}}$ ), and the higher $T_{\mathrm{g}}$ locates at almost the same temperature of the bulk PDLLA. As a lower $T_{\mathrm{g}}$ indicates higher segmental mobility, the results lead to a natural explanation of a two-layer model, where the lower $T_{\mathrm{g}}$ comes 
from a "mobile" interface layer and the higher $T_{\mathrm{g}}$ is caused by the bulk-like core polymer, similar to the previous reports $^{17,18,24,25}$.

\subsection{Segmental Relaxation Time.}
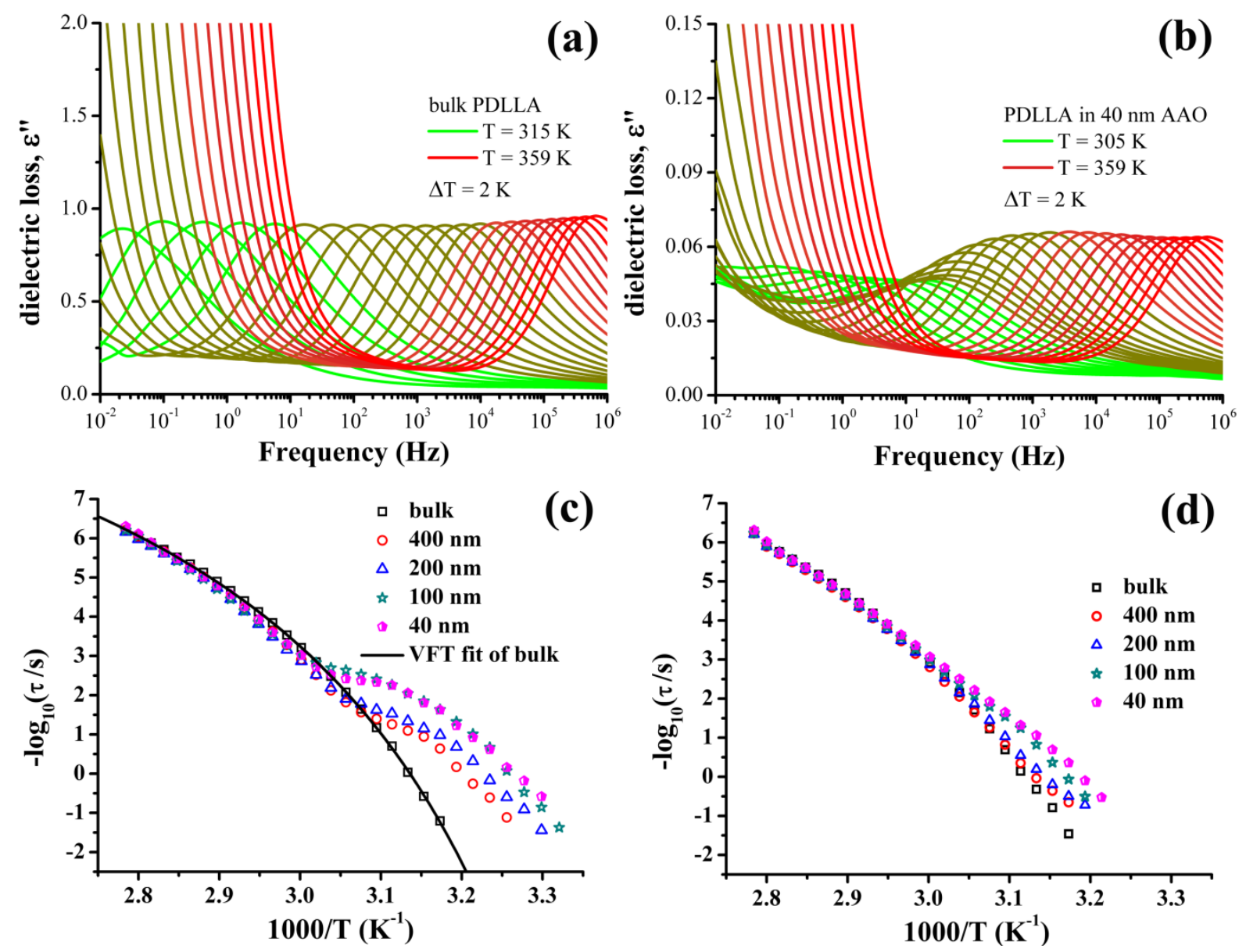

Figure 3. Typical curves of dielectric loss ( $\varepsilon$ ") as a function of frequency of bulk PDLLA (a) and infiltrated PDLLA in $40 \mathrm{~nm}$ AAO templates (b) with a temperature protocol 1 (P1) and the Arrhenius plot of bulk and infiltrated PDLLA with thermal protocols: (c) P1; (d) P2. The temperature interval of the curves in (a) and (b) was $2 \mathrm{~K}$.

To investigate in more detail the segmental dynamics of PDLLA, frequency scans were carried out. Figures 3a and 3b show the typical frequency plots of dielectric loss for bulk and infiltrated PDLLA at different temperatures. For bulk PDLLA, a single dielectric loss peak is seen. With increasing temperature, the peak position moves to higher frequencies, indicating shorter relaxation time. For infiltrated PDLLA, a similar shift of the loss peaks is seen. However, there is an increase in the intensity of the loss peak during heating. The physical origin of the increase will be discussed later. To extract the relaxation time, the phenomenological Havriliak-Negami (HN) function was applied to fit the dielectric loss curve: ${ }^{36}$ 


$$
\varepsilon^{*}(\omega)=\varepsilon^{\infty}+\frac{\Delta \varepsilon}{\left[1+(i \omega \tau)^{a}\right]^{b}}+\frac{\sigma_{c}}{i \varepsilon_{0} \omega}
$$

where the $\varepsilon^{\infty}$ is the dielectric permittivity in the absence of polarization processes (high-frequency limit), $\Delta \varepsilon, \tau, a$, $b$ represent the dielectric strength, relaxation time and shape parameters, respectively. The term on the right indicates the contribution of conduction, where $\varepsilon_{0}$ represents the vacuum permittivity.

It is noted that although the two loss peaks were observed during heating (Figure 2) only one relaxation peak is observed in the frequency scan, and the result can be nicely fitted with a single HN function. Similar treatments have been reported previously. ${ }^{25,37}$ The obtained $\tau$ should be regarded as an average of all the segments within the system. The characteristic relaxation time is plotted as a function of reciprocal temperature (Arrhenius plot). It turns out that the segmental dynamics of confined polymer deviates from the bulk polymer and thermal history plays a significant role. As shown in Figure 3c, with the thermal protocol P1, the bulk PLLA exhibits typical non-equilibrium behavior and can be fitted with the Vogel-Fulcher-Tammann (VFT) equation:38-40

$$
\tau_{\alpha}=\tau_{\infty} \exp \left(\frac{D_{T} T_{0}}{T-T_{0}}\right)
$$

where $\tau_{\infty}, D_{\mathrm{T}}$, and $T_{0}$ are constants to fit the data. An important observation in Figure 3c is the superposition of the curves at high temperatures and the obvious deviation at low temperatures. The transition temperature is 325-329 K. The difference in relaxation time becomes more obvious when the PDLLA was infiltrated in smaller pores. On the other hand, as shown in Figure 3d, for thermal protocol P2, the deviation of the segmental relaxation time is less pronounced as compared with that for P1, although the mobility of segments is still higher under confinement. It is striking to observe a strong "hysteresis" effect of the relaxation time of PDLLA under confinement.

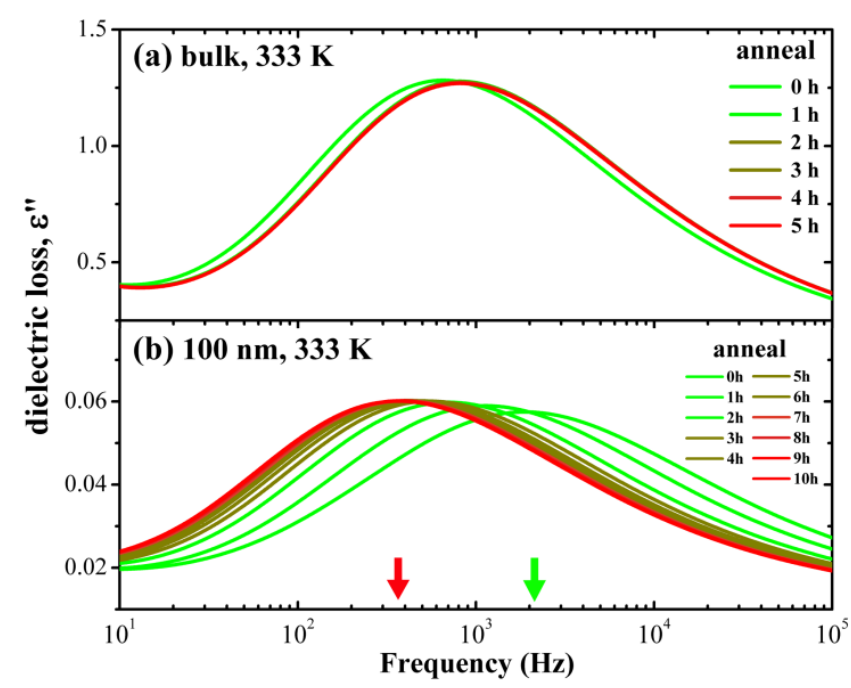

Figure 4. Dielectric loss ( $\varepsilon ")$ as a function of the frequency of bulk PDLLA (a) and infiltrated PDLLA in $100 \mathrm{~nm}$ 
AAO templates. The samples were annealed at $333 \mathrm{~K}$ for different periods.

If the sample is re-measured during heating right after the measurement following the protocol P2, the relaxation times are the same for the two measurements (Figure S2, Supporting Information). Therefore, it is very likely that the "hysteresis" results from kinetic reasons. This assignment can be further confirmed by Figure 4 . The measurement is designed as follows: first, the thermal history was erased by heating to $473 \mathrm{~K}$, then the sample was cooled down to $213 \mathrm{~K}$ at a rate of $20 \mathrm{~K} / \mathrm{min}$. Then, the sample was heated to a temperature at a rate of $20 \mathrm{~K} / \mathrm{min}$ to carry out the isothermal frequency scan. To access the kinetics, a narrower frequency range was chosen $1 \sim 10^{6} \mathrm{~Hz}$, thus each measurement needs only $77 \mathrm{~s}$. Figure 4 compares the change of dielectric loss curves with annealing at the same temperature. No significant change can be observed for the bulk sample. Interestingly, the peak maximum shifts towards lower frequencies with annealing time, indicating an increase of the relaxation time (up to 6 times). This clearly indicates that (part of) the PDLLA chains cooled from the melt rapidly as described by thermal protocol P1 are not in stable states, and change their physical configurations. Note that the hysteresis phenomena were reproduced after several times of cyclic measurement, indicating no chemical change has occurred during the measurement (Figure S3, Supporting Information).

The height of the dielectric loss peak is proportional to the number of dipoles for relaxation. As a qualitative analysis, the intensity of the peak was plotted as a function of temperature for both of the two thermal protocols (P1 and P2) in Figure S4 (Supporting Information). During heating or cooling of the bulk PDLLA, the $\Delta \varepsilon$ is almost independent of temperature. However, a transition-like increase of the $\Delta \varepsilon$ was observed for $100 \mathrm{~nm}$ PDLLA during heating according to $\mathrm{P} 1$. The temperature of this transition is $330 \mathrm{~K}$, which agrees with the endpoint of the $T_{\mathrm{g}}$ range.

The kinetics of the annealing process is expected to be temperature-dependent. The segmental relaxation time ( $\tau$ ) obtained by fitting the loss factor-temperature curve with HN function was plotted as a function of annealing time (Figure 5a and Figure S5). It is seen that the segmental relaxation time increases rapidly at first and then slowly upon further annealing. It is interesting to find that the segmental relaxation time $(\tau)$ can be described by a saturating exponential function of the type:

$$
\tau(\mathrm{t})=\tau_{\infty}-\left(\tau_{\infty}-\tau_{0}\right) * \exp \left(-\frac{t}{\tau_{c}}\right)
$$

where $\tau_{\infty}$ is the segmental relaxation time with infinite annealing time, $\tau_{0}$ is the initial relaxation time, and $\tau_{\mathrm{c}}$ is the characteristic time to describe the speed of approaching $\tau_{\infty}$. Obviously, when $t=0$, the exponential term is unity and $\tau$ equals its initial value $\tau_{0}$. When $t>>\tau_{\mathrm{c}}$, the exponential term vanishes, and $\tau$ approaches $\tau_{\infty}$. Note that the chosen 
equation is an approximation of the analytical expression reported previously. ${ }^{41}$

Figure $5 \mathrm{~b}$ represents plotted the normalized segmental relaxation time as a function of the annealing time, and it is clear that the segmental relaxation approaches a stable value faster at higher temperatures. Interestingly, the logarithm of $\tau_{\mathrm{c}}$ increases linearly with the inverse temperature, indicating a typical Arrhenius behavior:

$$
\tau_{c}=A \exp \left(\frac{E_{a}}{R T}\right)
$$

where $A$ is a constant, $E_{\mathrm{a}}$ is the activation energy, and $R$ is the universal gas constant. The $E_{\mathrm{a}}$ is estimated by the slope of the fitting curve, yielding a value of $282 \pm 5 \mathrm{~kJ} / \mathrm{mol}$. This value is remarkably higher than the activation energy of adsorption of PS on silicon oxide $(66 \pm 11 \mathrm{~kJ} / \mathrm{mol})$ which has been attributed to local noncooperative rearrangements. ${ }^{42}$ Such a high activation energy probably indicates a cooperative nature of the relaxation process. The data points in Figure 5c and Figure 3c were plotted together in Figure S6 in the Supporting Information. It was found that the equilibration process can be superimposed onto the segmental relaxation by multiplying a temperatureindependent constant. For the investigated system, the equilibration or adsorption process is $10^{7}$ times lower than the segmental mobility. Therefore, the picture of molecular process of adsorption could be that after many times $\left(\sim 10^{7}\right)$ of trials, new chains are adsorbed and the interfacial free volume is thus annihilated, in line with the mechanism described in thin films. ${ }^{43}$ Furthermore, we noticed that the $\left(\tau_{\infty}-\tau_{0}\right) / \tau_{\infty}$, characterizing the "degree of deviation from equilibrium”, decreases with temperature (Figure 5d). 
(a)
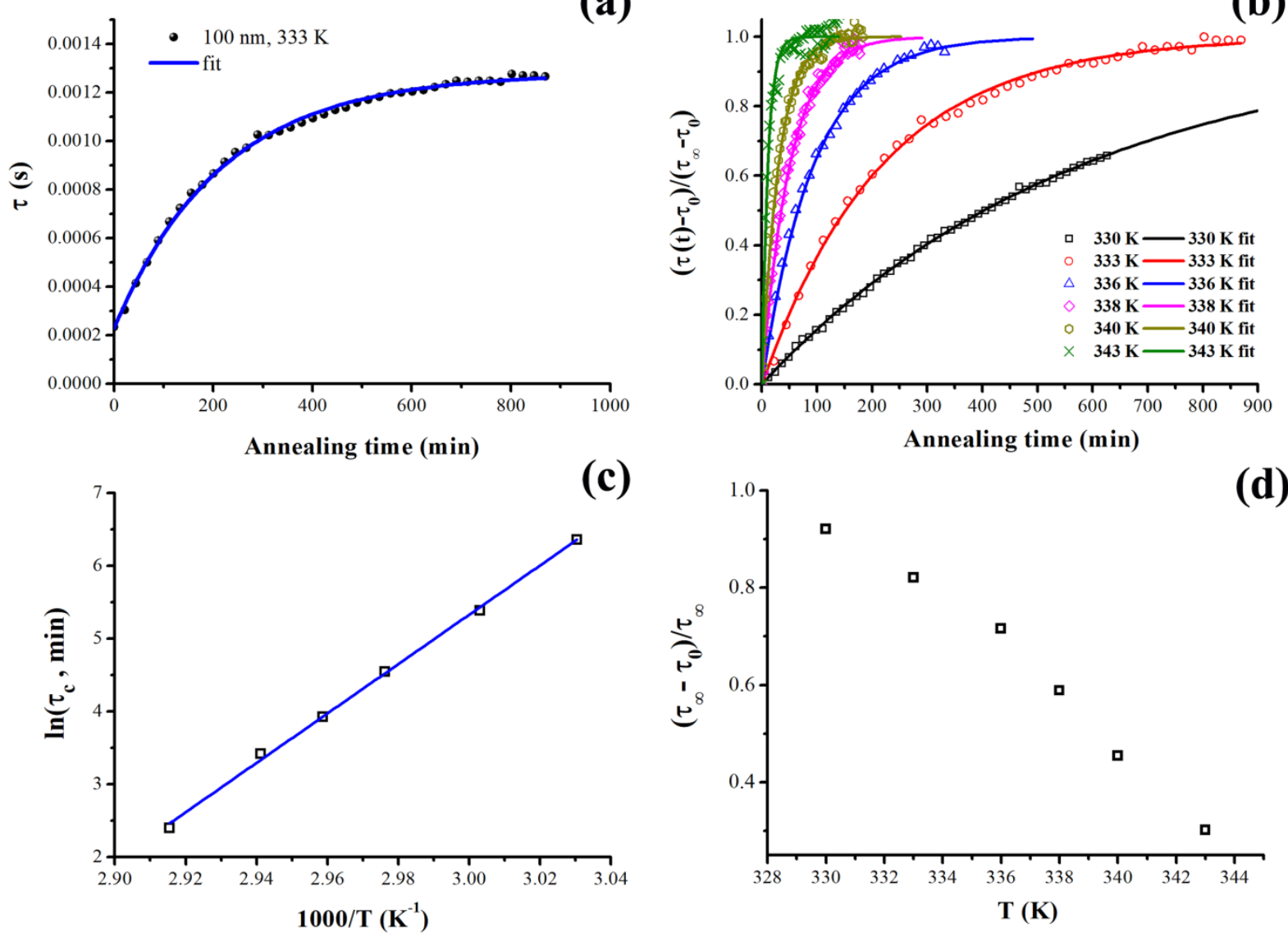

Figure 5. (a) Change of the segmental relaxation time $(\tau)$ as a function of annealing time at $333 \mathrm{~K}$; (b) normalized segmental relaxation time, $\frac{\tau_{\max }(t)-\tau_{0}}{\tau_{\infty}-\tau_{0}}$, as a function of annealing time of infiltrated PDLLA in $100 \mathrm{~nm}$ AAO at different temperatures; (c) Arrhenius plot of the characteristic time $\tau_{\mathrm{c}} \sim 1000 / \mathrm{T}$. (d) change of $\left(\tau_{\infty}-\tau_{0}\right) / \tau_{\infty}$ as a function of temperature. The sample is infiltrated PDLLA within $100 \mathrm{~nm}$ AAO templates.

\subsection{Physical Origin of the Change of Segmental Dynamics.}

The effect of the interface on segmental dynamics has been frequently interpreted considering the counterbalance between confinement and adsorption effects. A faster dynamics was observed in poly(methyl phenyl siloxane) (PMPS) under confinement within nanoporous glasses with pore sizes range from $2.5 \mathrm{~nm}$ to $20 \mathrm{~nm} .{ }^{44,45} \mathrm{~A}$ transition from VFT-like to Arrhenius law was observed when the pore size decreased to $5 \mathrm{~nm}$. Deviations of bulk dynamics at low temperatures were also reported in PPG confined in AAO templates. ${ }^{24,25}$

The confinement effect is frequently discussed in terms of the cooperative rearrangement region (CRR) model proposed by Adam and Gibbs. ${ }^{46}$ The model argued that the CRR size ( $\left.\xi\right)$ became larger with decreasing temperature and the increasing $\xi(T)$ attributes the increase of segmental relaxation time. It is estimated that $\xi$ is in the range of several nanometers at $T_{\mathrm{g}},{ }^{47,}{ }^{48}$ When the domain size is small, the $\xi$ cannot exceed the domain size, therefore the 
segmental mobility will be enhanced. ${ }^{3,49}$ While this picture is straightforward and attractive, the size of AAO in our study is much bigger than $\xi$. The deviation of VFT equation is prominent even for $400 \mathrm{~nm}$ AAO in thermal history P1, thus this explanation can be excluded.

Chain segments in proximity to the surface possess higher freedom to move, i.e., interfacial free volume, thus would have shorter relaxation time. ${ }^{8-10}$ The surface to volume ratio increases with the decreasing pore diameter in AAO pores, which agrees with the stronger deviation of bulk dynamics in Figure 3c. The thermal history dependence of the relaxation time indicates the non-equilibrium nature of the quenched sample. During annealing, the excess in segmental mobility gets reduced (Figure 5a). Thermal history P2, with prolonged time in the melt, approaches closer to bulk behavior (Figure 3d).

The equilibration process was proposed to be governed by irreversible adsorption in polymer thin films. ${ }^{43}$ Intuitively, the physical mechanism in question should allow the reduction of free energy by reduction of free volume near the interface. The characteristic time to reach equilibrium $\left(10^{3} \sim 10^{4} \mathrm{~s}\right)$ is $\sim 7$ orders of magnitude longer than the segmental relaxation time $\left(10^{-4} \sim 10^{-3} \mathrm{~s}\right)$. The ratio of characteristic time agrees with the experiments on thin films. ${ }^{43}$ It is reasonable to see that the value of $\left(\tau_{\infty}-\tau_{0}\right) / \tau_{\infty}$ of PDLLA changes significantly with temperature, which agrees with the mechanism of reversible adsorption/desorption of chains. The equilibrium constant moves in favor of desorption at elevated temperatures.

\subsection{Impact of Segmental Dynamics on Crystallization.}

It is interesting to consider the relationship between segmental dynamics and the cold crystallization of PLLA. In our previous paper ${ }^{31}$, the cold crystallization of infiltrated PLLA within nanoporous AAO was enhanced. The temperature scan rate of DSC is much higher than that of DRS. Therefore, in this case, polymer chains have less time to adsorb. It is expected that the segmental mobility is enhanced as compared with the bulk PLLA. Therefore, the unusual enhanced nucleation effect of the amorphous AAO wall may be related to the mobile chains in the vicinity of the wall. According to the classical formula proposed by Turnbull and Fisher ${ }^{50}$, the polymer nucleation rate is given by:

$$
I=I_{0} \exp \left(-\frac{\Delta G^{*}+\Delta E}{k T}\right)
$$

where $\Delta G^{*}$ is the free energy barrier of forming a critical nucleus and $\Delta E$ is the activation energy of diffusion of segments across the phase boundary. Intuitively, the term $\Delta G^{*}$ should be independent on the confinement if we assume homogeneous nucleation. However, the term $\Delta E$ is directly related to the chain mobility. Higher mobility will 
reduce the nucleation barrier and contribute to a higher nucleation rate.

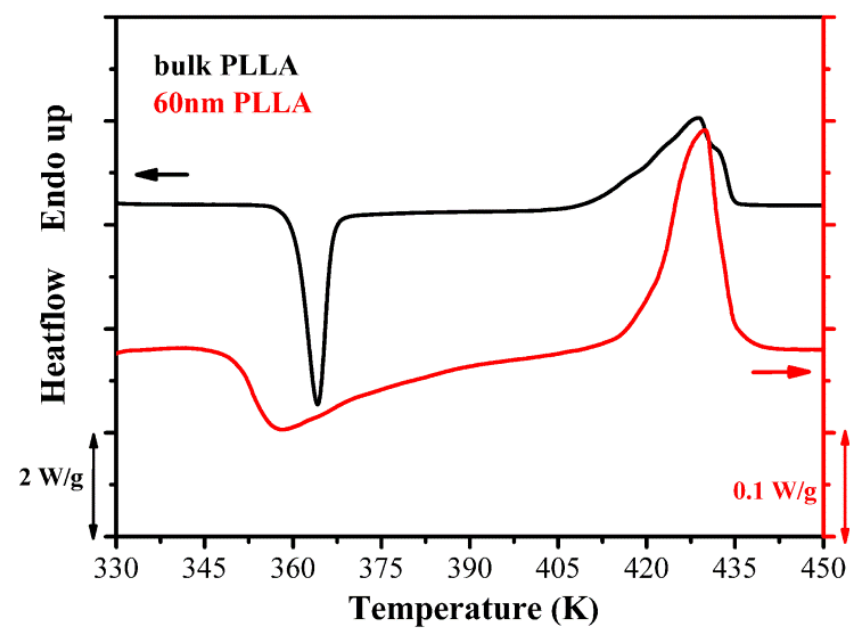

Figure 6. DSC heating scans of bulk and infiltrated PLLA from the glassy state after quenched under the condition of $100 \mathrm{~K} / \mathrm{min}$. The heating rate is $10 \mathrm{~K} / \mathrm{min}$.

As the segmental mobility "slows down" during annealing, it is interesting to see how the cold crystallization behavior changes with the thermal treatment. Figure 6 shows the DSC heating curves of quenched PLLA bulk and confined samples. The cold crystallization peak of infiltrated PLLA is broader than that of the bulk PLLA. The peak temperature of cold crystallization $\left(T_{\mathrm{cc}}\right)$ of infiltrated PLLA is lower than that of the bulk PLLA, which agrees with our previous study. ${ }^{31}$ To understand the difference in curve shape, we need to consider the difference in the crystallization process of bulk and confined samples. In bulk, once nucleation starts, the crystallization will proceed quickly via crystal growth. On the other hand, the nuclei can only influence the crystallization of a single pore. The pores without nuclei will have to "wait" for the formation of nuclei. Because homogeneous nucleation is a random process, nuclei may form quickly in some pores, while it may take a long time to form a nucleus in some others pores. This is the reason why the crystallization exotherm is much broader for polymers in AAO.

To explore how the chain adsorption can influence the cold crystallization of PLLA, the samples were cooled from the melt at a cooling rate of $100 \mathrm{~K} / \mathrm{min}$ to $342 \mathrm{~K}$, and they were annealed at that temperature for different periods. With increasing annealing time, the $T_{\mathrm{cc}}$ of bulk PLLA shifts to lower temperatures and the area of the cold crystallization peak becomes smaller, indicating crystallization during annealing (Figure 7a). The crystals formed during annealing act as nuclei for further crystallization during heating. Unlike bulk PLLA, the cold crystallization peak of infiltrated PLLA moves to higher temperatures (Figure 7b), although crystallization also occurs in infiltrated PLLA during annealing as shown by GIWAXS (Figure S7).

Figure 7c shows the change of $T_{\mathrm{cc}}$ as a function of annealing time. When the annealing time reaches $60 \mathrm{~min}$, the 
$T_{\mathrm{cc}}$ of infiltrated PLLA reaches $381 \mathrm{~K}$, which is $20 \mathrm{~K}$ higher than the initial value (Figure 7c). This observation agrees with our expectation that adsorbed chains restrict the crystallization of PLLA. The annealing temperature selected, $342 \mathrm{~K}$, is above the $T_{\mathrm{g}}$. Therefore, crystallization will occur during annealing. As shown in Figure $7 \mathrm{~d}$, the crystallization enthalpy during annealing can be obtained by $\Delta H_{\mathrm{m}}-\Delta H_{\mathrm{cc}}$. For bulk PLLA, the degree of crystallization increases slowly at the beginning and then rapidly during annealing. No cold crystallization peak can be detected for bulk PLLA after annealing for $30 \mathrm{~min}$, indicating that crystallization has reached saturation during annealing. However, for the infiltrated PLLA in AAO pores, the degree of crystallinity is higher than that of the bulk for short periods of annealing but increases slowly. A maximum value of 0.65 is reached after annealing for 25 min. Further annealing does not contribute to higher crystallinity.

The different cold crystallization behavior of bulk and infiltrated PLLA can be explained by the interplay between interfacial enhanced crystallization and adsorption. As shown in Figure 5, adsorption is a gradual process. For short-time annealing, the chains may not have enough time to adsorb on the AAO wall. Those chains with higher mobility will crystallize faster than in the bulk during annealing. With prolonged annealing, those uncrystallized PLLA chains will have enough time for adsorption. Once adsorbed, the nucleation rate of those chains decreased significantly and the cold crystallization peak shifts towards higher temperatures.
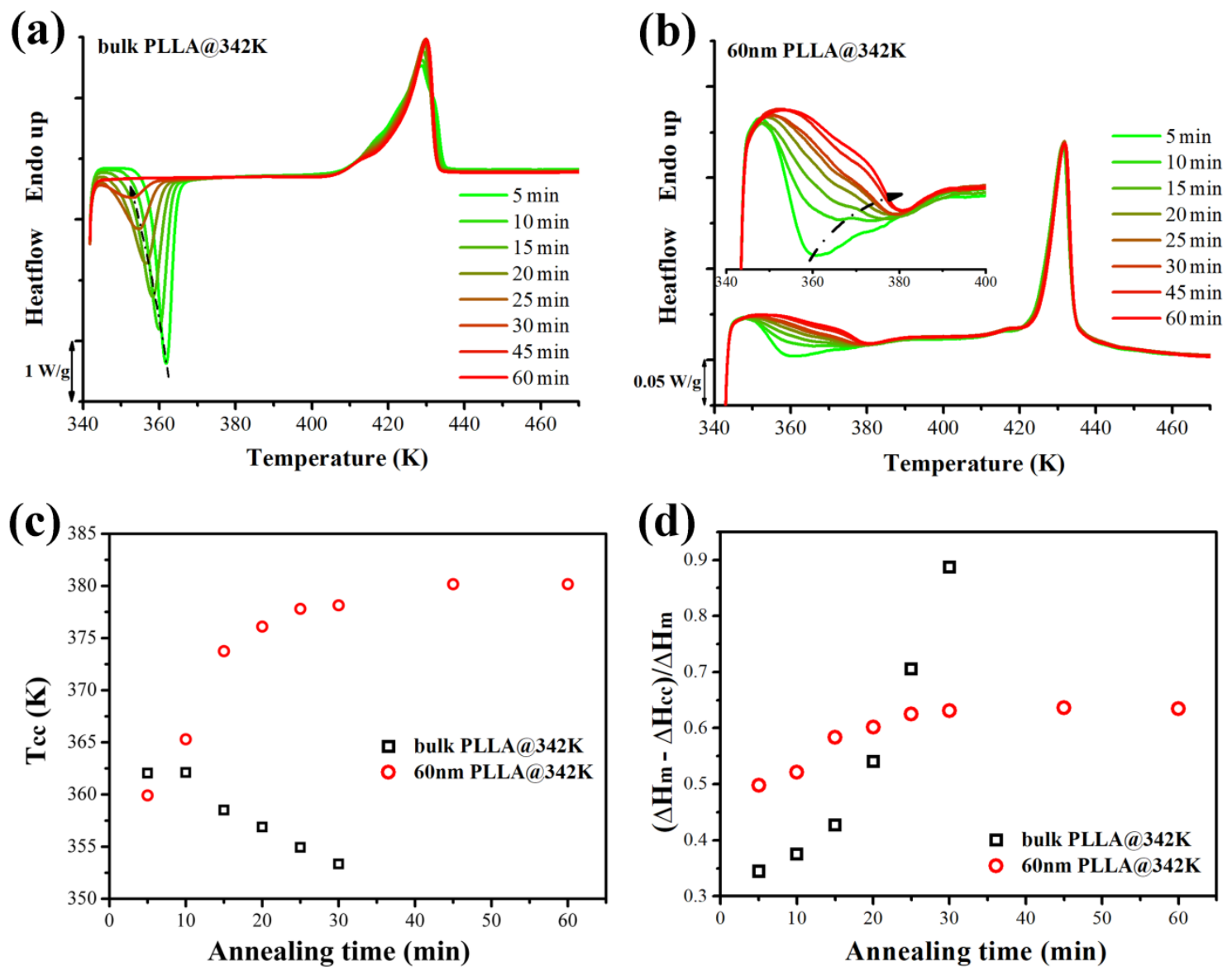
Figure 7. DSC heating curves of quenched bulk (a) and infiltrated PLLA (b) in $60 \mathrm{~nm}$ AAO annealed at $342 \mathrm{~K}$ for different periods. The $T_{\mathrm{cc}}(\mathrm{c})$ and enthalpy (d) during annealing at $342 \mathrm{~K}$ extracted from DSC measurements.

\section{CONCLUSIONS}

We investigated the segmental dynamics of PDLLA within nonporous alumina templates with different pore diameters. The first “enhanced” and gradual "slowdown” of segmental mobility upon annealing at temperatures near the glass transition was observed. The deviation from bulk behavior was more pronounced for PDLLA in smaller AAO pores. The non-equilibrium feature of the segmental mobility was shown to originate from the reversible adsorption of the PDLLA chains: surface effect caused the "speed up" and subsequent "time-consuming” adsorption attributed to the "slowdown" of segmental dynamics. The rate of chain adsorption exhibited activation energy of 282 $\pm 5 \mathrm{~kJ} / \mathrm{mol}$, corresponding to a cooperative nature of the relaxation process. The observations of this work explain the enhanced cold crystallization of PLLA from the glassy state ${ }^{31}$ by the enhanced mobility of chain segments in the vicinity of the AAO wall (surface nucleation). The correlation between chain adsorption and crystallization was further confirmed by the observation that cold crystallization is highly restricted when the chains are adsorbed onto the AAO wall after annealing. Our extensive experimental data provide a direct relationship between the segmental dynamics and crystallization in 2D confined environments.

\section{ACKNOWLEDGEMENTS}

This work is supported by the National Key R\&D Program of China (2017YFE0117800) and the National Natural Science Foundation of China (21873109, 51820105005). G. L. is grateful to the Youth Innovation Promotion Association of the Chinese Academy of Sciences (2015026) and the Open Research Fund of State Key Laboratory of Polymer Physics and Chemistry (CIAC) (201510). A. J. M. gratefully acknowledges financial support from MINECO through project MAT2017-83014-C2-1-P. G. L., D. W., and A. J. M. acknowledge European funding by the RISE BIODEST project (H2020-MSCA-RISE-2017-778092). G. S. thanks Dr. Wensheng Bu for assistance with the DRS measurement.

\section{SUPPORTING INFORMATION}

Dielectric loss for empty AAO templates, segmental relaxation time of infiltrated PDLLA with thermal protocol P2 and during subsequent heating process, segmental relaxation time of infiltrated PDLLA with thermal protocol P1, P2 and repeated P1, dielectric strength of bulk and infiltrated PDLLA, changes of segmental relaxation time of infiltrated PDLLA during annealing, comparison of the characteristic times in Figure 5c and Figure 3c, GIWAXS of infiltrated 
PLLA.

\section{REFERENCES}

1. Mataz, A.; Gregory, B. M. Effects of confinement on material behaviour at the nanometre size scale. J. Phys.: Condens. Matter 2005, 17 (15), R461.

2. Napolitano, S.; Capponi, S.; Vanroy, B. Glassy dynamics of soft matter under 1D confinement: How irreversible adsorption affects molecular packing, mobility gradients and orientational polarization in thin films. Eur. Phys. J. 2013, 36 (6), 61.

3. Forrest, J. A.; Dalnoki-Veress, K. The glass transition in thin polymer films. Adv. Colloid Interface Sci. 2001, 94 (13), 167-195.

4. Spiece, J.; Martinez-Tong, D. E.; Sferrazza, M.; Nogales, A.; Napolitano, S. Are polymers glassier upon confinement? Soft Matter 2015, 11 (31), 6179-6186.

5. Fragiadakis, D.; Pissis, P.; Bokobza, L. Glass transition and molecular dynamics in poly (dimethylsiloxane)/silica nanocomposites. Polymer 2005, 46 (16), 6001-6008.

6. Salaniwal, S.; Kumar, S. K.; Douglas, J. F. Amorphous solidification in polymer-platelet nanocomposites. Phys. Rev. Lett. 2002, 89 (25), 258301.

7. Gao, Y.-Y.; Hu, F.-Y.; Liu, J.; Wang, Z. Molecular dynamics simulation of the glass transition temperature of fullerene filled cis-1,4-polybutadiene nanocomposites. Chin. J. Polym. Sci. 2018, 36 (1), 119-128.

8. Fakhraai, Z.; Forrest, J. A. Measuring the Surface Dynamics of Glassy Polymers. Science 2008, 319 (5863), $600-604$.

9. Paeng, K.; Swallen, S. F.; Ediger, M. D. Direct Measurement of Molecular Motion in Freestanding Polystyrene Thin Films. J. Am. Chem. Soc. 2011, 133 (22), 8444-8447.

10. Pye, J. E.; Rohald, K. A.; Baker, E. A.; Roth, C. B. Physical Aging in Ultrathin Polystyrene Films: Evidence of a Gradient in Dynamics at the Free Surface and Its Connection to the Glass Transition Temperature Reductions. Macromolecules 2010, 43 (19), 8296-8303.

11. Reiter, G. Dewetting as a Probe of Polymer Mobility in Thin Films. Macromolecules 1994, 27 (11), $3046-3052$.

12. Rotella, C.; Wübbenhorst, M.; Napolitano, S. Probing interfacial mobility profiles via the impact of nanoscopic confinement on the strength of the dynamic glass transition. Soft Matter 2011, 7 (11), 5260-5266.

13. Papon, A.; Montes, H.; Hanafi, M.; Lequeux, F.; Guy, L.; Saalwächter, K. Glass-Transition Temperature Gradient in Nanocomposites: Evidence from Nuclear Magnetic Resonance and Differential Scanning Calorimetry. Phys.Rev. Lett. 2012, 108 (6), 065702.

14. Krutyeva, M.; Wischnewski, A.; Monkenbusch, M.; Willner, L.; Maiz, J.; Mijangos, C.; Arbe, A.; Colmenero, J.; Radulescu, A.; Holderer, O.; Ohl, M.; Richter, D. Effect of Nanoconfinement on Polymer Dynamics: Surface Layers and Interphases. Phys. Rev. Lett. 2013, 110 (10), 108303.

15. Shin, K.; Obukhov, S.; Chen, J. T.; Huh, J.; Hwang, Y.; Mok, S.; Dobriyal, P.; Thiyagarajan, P.; Russell, T. P. Enhanced mobility of confined polymers. Nat. Mater. 2007, 6 (12), 961-965.

16. Blaszczyk-Lezak, I.; Hernández, M.; Mijangos, C. One Dimensional PMMA Nanofibers from AAO Templates. Evidence of Confinement Effects by Dielectric and Raman Analysis. Macromolecules 2013, 46 (12), 4995-5002.

17. Li, L.; Zhou, D.; Huang, D.; Xue, G. Double Glass Transition Temperatures of Poly(methyl methacrylate) Confined in Alumina Nanotube Templates. Macromolecules 2014, 47 (1), 297-303.

18. Li, L.; Chen, J.; Deng, W.; Zhang, C.; Sha, Y.; Cheng, Z.; Xue, G.; Zhou, D. Glass Transitions of Poly(methyl methacrylate) Confined in Nanopores: Conversion of Three- and Two-Layer Models. J. Phys. Chem. B 2015, 119 (15), 5047-5054.

19. Wang, H.; Chang, T.; Li, X.; Zhang, W.; Hu, Z.; Jonas, A. M. Scaled down glass transition temperature in confined polymer nanofibers. Nanoscale 2016, 8 (32), 14950-14955.

20. Alexandris, S.; Papadopoulos, P.; Sakellariou, G.; Steinhart, M.; Butt, H.-J.; Floudas, G. Interfacial Energy and Glass 
Temperature of Polymers Confined to Nanoporous Alumina. Macromolecules 2016, 49 (19), 7400-7414.

21. Alexandris, S.; Sakellariou, G.; Steinhart, M.; Floudas, G. Dynamics of Unentangled cis-1,4-Polyisoprene Confined to Nanoporous Alumina. Macromolecules 2014, 47 (12), 3895-3900.

22. Reid, D. K.; Alves Freire, M.; Yao, H.; Sue, H.-J.; Lutkenhaus, J. L. The Effect of Surface Chemistry on the Glass Transition of Polycarbonate Inside Cylindrical Nanopores. ACS Macro Lett. 2015, 4 (2), 151-154.

23. Martin, J.; Mijangos, C.; Sanz, A.; Ezquerra, T. A.; Nogales, A. Segmental Dynamics of Semicrystalline Poly(vinylidene fluoride) Nanorods. Macromolecules 2009, 42 (14), 5395-5401.

24. Tarnacka, M.; Kaminski, K.; Mapesa, E. U.; Kaminska, E.; Paluch, M. Studies on the Temperature and Time Induced Variation in the Segmental and Chain Dynamics in Poly(propylene glycol) Confined at the Nanoscale. Macromolecules 2016, 49 (17), 6678-6686.

25. Talik, A.; Tarnacka, M.; Grudzka-Flak, I.; Maksym, P.; Geppert-Rybczynska, M.; Wolnica, K.; Kaminska, E.; Kaminski, K.; Paluch, M. The Role of Interfacial Energy and Specific Interactions on the Behavior of Poly(propylene glycol) Derivatives under 2D Confinement. Macromolecules 2018, 51 (13), 4840-4852.

26. Duran, H.; Gitsas, A.; Floudas, G.; Mondeshki, M.; Steinhart, M.; Knoll, W. Poly( $\gamma$-benzyl-l-glutamate) Peptides Confined to Nanoporous Alumina: Pore Diameter Dependence of Self-Assembly and Segmental Dynamics. Macromolecules 2009, 42 (8), 2881-2885.

27. Suzuki, Y.; Duran, H.; Akram, W.; Steinhart, M.; Floudas, G.; Butt, H. J. Multiple nucleation events and local dynamics of poly(epsilon-caprolactone) (PCL) confined to nanoporous alumina. Soft Matter 2013, 9 (38), 9189-9198.

28. Suzuki, Y.; Duran, H.; Steinhart, M.; Butt, H.-J.; Floudas, G. Homogeneous crystallization and local dynamics of poly(ethylene oxide) (PEO) confined to nanoporous alumina. Soft Matter 2013, 9 (9), 2621-2628.

29. Napolitano, S.; Glynos, E.; Tito, N. B. Glass transition of polymers in bulk, confined geometries, and near interfaces. Rep. Prog. Phys. 2017, 80 (3), 036602.

30. Serghei, A.; Chen, D.; Lee, D. H.; Russell, T. P. Segmental dynamics of polymers during capillary flow into nanopores. Soft Matter 2010, 6 (6), 1111-1113.

31. Guan, Y.; Liu, G.; Ding, G.; Yang, T.; Mueller, A. J.; Wang, D. Enhanced Crystallization from the Glassy State of Poly(L-lactic acid) Confined in Anodic Alumina Oxide Nanopores. Macromolecules 2015, 48 (8), $2526-2533$.

32. Shi, G.; Liu, G.; Su, C.; Chen, H.; Chen, Y.; Su, Y.; Müller, A. J.; Wang, D. Reexamining the Crystallization of Poly(عcaprolactone) and Isotactic Polypropylene under Hard Confinement: Nucleation and Orientation. Macromolecules 2017, 50 (22), 9015-9023.

33. Su, C.; Shi, G.; Wang, D.; Liu, G. A Model for the Crystal Orientation of Polymers Confined in 1D Nanocylinders. Acta Polym. Sin. 2019, 50 (3), 281-290.

34. Ren, J.; Urakawa, O.; Adachi, K. Dielectric and Viscoelastic Studies of Segmental and Normal Mode Relaxations in Undiluted Poly(d,l-lactic acid). Macromolecules 2003, 36 (1), 210-219.

35. Ren, J.; Adachi, K. Dielectric Relaxation in Blends of Amorphous Poly(dl-lactic acid) and Semicrystalline Poly(llactic acid). Macromolecules 2003, 36 (14), 5180-5186.

36. Havriliak, S.; Negami, S. A complex plane representation of dielectric and mechanical relaxation processes in some polymers. Polymer 1967, 8, 161-210.

37. Tarnacka, M.; Talik, A.; Kamińska, E.; Geppert-Rybczyńska, M.; Kaminski, K.; Paluch, M. The Impact of Molecular Weight on the Behavior of Poly(propylene glycol) Derivatives Confined within Alumina Templates. Macromolecules 2019, 52 (9), 3516-3529.

38. Vogel, H. Das Temperaturabhangigkeitsgesetz der Viskositat von Flussigkeiten. Phys. Z. 1921, 22, 645-646.

39. Fulcher, G. S. Analysis of recent measurements of the viscosity of glasses. J. Am. Ceram. Soc. 1925, 8 (6), 339-355.

40. Tammann, G.; Hesse, W. Die Abhängigkeit der Viscosität von der Temperatur bie unterkühlten Flüssigkeiten. Z. Anorg. Allg. Chem. 1926, 156 (1), 245-257.

41. Vanroy, B.; Wübbenhorst, M.; Napolitano, S. Crystallization of thin polymer layers confined between two adsorbing walls. ACS Macro Lett. 2013, 2 (2), 168-172. 
42. Housmans, C.; Sferrazza, M.; Napolitano, S. Kinetics of Irreversible Chain Adsorption. Macromolecules 2014, 47 (10), 3390-3393.

43. Panagopoulou, A.; Napolitano, S. Irreversible Adsorption Governs the Equilibration of Thin Polymer Films. Phys. Rev. Lett. 2017, 119 (9), 097801.

44. Schönhals, A.; Goering, H.; Schick, C.; Frick, B.; Zorn, R. Glass transition of polymers confined to nanoporous glasses. Colloid Polym. Sci. 2004, 282 (8), 882-891.

45. Schönhals, A.; Goering, H.; Schick, C.; Frick, B.; Zorn, R. Polymers in nanoconfinement: What can be learned from relaxation and scattering experiments? J. Non-Cryst. Solids 2005, 351 (33), 2668-2677.

46. Adam, G.; Gibbs, J. H. On the Temperature Dependence of Cooperative Relaxation Properties in Glass-Forming Liquids. J. Chem. Phys. 1965, 43 (1), 139-146.

47. Kivelson, D.; Kivelson, S. A.; Zhao, X.; Nussinov, Z.; Tarjus, G. A thermodynamic theory of supercooled liquids. Physica A 1995, 219 (1), 27-38.

48. Fischer, E. W.; Donth, E.; Steffen, W. Temperature dependence of characteristic length for glass transition. Phys. Rev. Lett. 1992, 68 (15), 2344-2346.

49. Wübbenhorst, M.; Napolitano, S., Deviations from Bulk Glass Transition Dynamics of Small Molecule Glass Formers: Some Scenarios in Relation to the Dimensionality of the Confining Geometry. In Dynamics in Geometrical Confinement, Kremer, F., Ed. Springer International Publishing: Cham, 2014; pp 247-277.

50. Turnbull, D.; Fisher, J. C. Rate of Nucleation in Condensed Systems. J. Chem. Phys. 1949, 17 (1), $71-73$. 
For Table of Contents Use Only

Segmental Dynamics Govern the Cold Crystallization of Poly(lactic acid) in

\section{Nanoporous Alumina}

Guangyu Shi, Yu Guan, Guoming Liu*, Alejandro J. Müller, and Dujin Wang

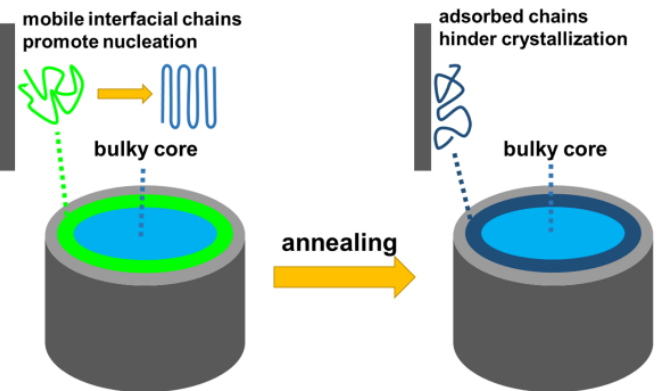

